\title{
Fibroelastosis pleuroparenquimatosa idiopática: reporte de caso
}

\author{
Idiopathic pleuroparenchymatous \\ fibroelastosis: a case report
}

\author{
Tatiana Suárez Poveda, MD ${ }^{1}$ Patricia Eugenia Gil-Serrano, MD² María Camila Arango, MD ${ }^{3}$ \\ VIANA PALACIO, MD ${ }^{4}$
}

\section{Resumen}

La fibroelastosis pleuroparenquimatosa idiopática (FPI) recientemente se describió como una causa de fibrosis pulmonar de rápida evolución y mal pronóstico. En este reporte describimos el caso de una paciente joven, previamente sana, quien se presentó al servicio de urgencias de nuestra institución con un cuadro de falla ventilatoria e imágenes compatibles con fibrosis pulmonar. Se llevó a lobectomía pulmonar, donde el estudio histopatológico confirmó el diagnóstico de fibroelastosis pleuroparenquimatosa idiopática. La paciente falleció antes de recibir un trasplante bipulmonar. La paciente tuvo una presentación usual clínica, por imágenes e histopatología de fibroelastosis pleuroparenquimatosa; tanto en la radiografía de tórax como en la tomografía computada de alta resolución se encontró pérdida de volumen pulmonar, cambios fibrocicatriciales de los lóbulos superiores, engrosamiento pleural y de septos interlobulillares. La patología evidenció marcado engrosamiento pleural con infiltrado inflamatorio linfocitario. En conclusión, la fibroelastosis pleuroparenquimatosa es una patología pulmonar fibrosante, recientemente descrita y de pobre pronóstico, en la cual no se han estandarizado los criterios diagnósticos ni el tratamiento. Por esto es importante diferenciarla de otras enfermedades intersticiales pulmonares.

Palabras clave: fibroelastosis pleuroparenquimatosa idiopática, enfermedad pulmonar intersticial, fibrosis pulmonar, pulmón.

\begin{abstract}
Idiopathic pleuroparenchymatous fibroelastosis is a recently reported cause of rapidly evolving pulmonary fibrosis and poor prognosis. We describe the case of a previously healthy, young patient who presented in our institution with ventilatory failure and images compatible with pulmonary fibrosis. Pulmonary lobectomy was performed, and histopathological studies confirmed idiopathic pleuroparenchymal fibroelastosis. The patient died before bilateral lung transplant was performed. Clinical, imaginologic, and histopathologic presentation of our case of idiopathic pleuroparenchymal fibroelastosis was usual, chest radiograph and high resolution computed tomography showed volume loss, upper lobes fibrocicatricial changes, pleural and interlobular septal thickening. Pathology showed extensive pleural thickening with inflammatory lymphocytic infiltrate. In conclusion, idiopathic pleuroparenchymal fibroelastosis is a recently described fibrosing lung disease, with poor prognosis, and without standardized diagnostic criteria and treatment. This is why it is important to differentiate it from other interstitial lung diseases.
\end{abstract}

Keywords: idiopathic pleuroparenchymatous fibroelastosis, interstitial lung disease, pulmonary fibrosis, lung.
${ }^{1}$ Médica Radióloga. Especialista en Ra-
diología de Trauma y Urgencias. Hospi-
tal Universitario San Vicente Fundación.
Medellín, Colombia.
${ }^{2}$ Médica Residente de Radiología, Uni-
versidad CES. Medellín, Colombia.
${ }^{3}$ Médica Residente de Radiología, Univer-
sidad de Antioquia. Medellín, Colombia.
${ }^{4}$ Médica Residente de Radiología, Univer-
sidad de Antioquia. Medellín, Colombia.
Autor de correspondencia
Patricia Eugenia Gil Serrano. Diagonal
23 \# 18B-132. Tel.: +57 (318)529.86.76.
Correo electrónico: patriciagil16@hot-
mail.com.
Recibido: 11/11/17. Aceptado: 12/12/17. 


\section{Introducción}

La fibroelastosis pleuroparenquimatosa idiopática es una neumonía intersticial crónica caracterizada por el engrosamiento de fibras elásticas de la pleura y del parénquima subpleural, que compromete principalmente los lóbulos superiores. Esta entidad se mencionó por primera vez en la literatura médica por Frankel y colaboradores en el año 2004, aunque el mismo concepto designado como fibrosis pulmonar idiopática del lóbulo superior fue propuesto en 1992 por Aminati y colaboradores; sin embargo, en el año 2013, la fibroelastosis pleuroparenquimatosa se incluyó en la clasificación de neumonías intersticiales idiopáticas raras por la ATS/ERS (American Thoracic Society/European Respiratory Society) con 120 casos publicados hasta el momento en la literatura a nivel mundial (1-5).

\section{Presentación del caso}

Paciente femenina de 35 años, sin antecedentes patológicos de importancia conocidos. Ingresó al servicio de urgencias por cuadro clínico de 1 mes de evolución, consistente en dolor torácico pleurítico, disnea, tos seca, pérdida de peso $(8 \mathrm{~kg})$ y dolor en hipocondrio derecho. Se realizó radiografía de tórax que mostró opacidades reticulares, por lo que se llevó a tomografía axial computada de alta resolución (TACAR) para una mejor caracterización del hallazgo radiográfico, evidenciando pérdida de volumen en los lóbulos superiores con distorsión de la arquitectura y engrosamiento septal por cambios fibróticos, así como engrosamiento pleural apical bilateral (Figura 1), pleural y de septos interlobulillares

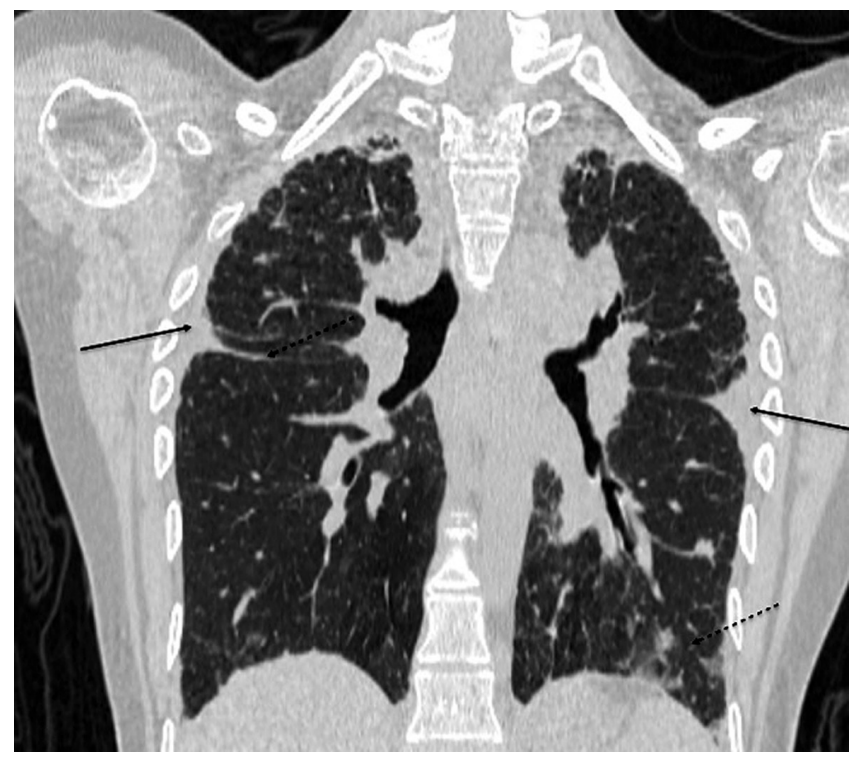

Figura 1. Tomografía de tórax de alta resolución, reconstrucción coronal. Pérdida de volumen en los lóbulos superiores con distorsión de la arquitectura y engrosamiento septal por cambios fibróticos (flecha discontinua) y engrosamiento pleural apical bilateral (flechas).

de predominio en lóbulos superiores (Figura 2A) asociado con consolidaciones subpleurales de influencia en lóbulos superiores y distorsión de la arquitectura por cambios fibróticos (Figura 2B). Se llevó a lobectomía segmentaria por videotoracoscopia donde se evidenció proceso fibrótico del parénquima pulmonar.

La patología evidenció marcado engrosamiento pleural con infiltrado inflamatorio linfocitario y fibrosis, la cual se extendía al parénquima cicatricial con
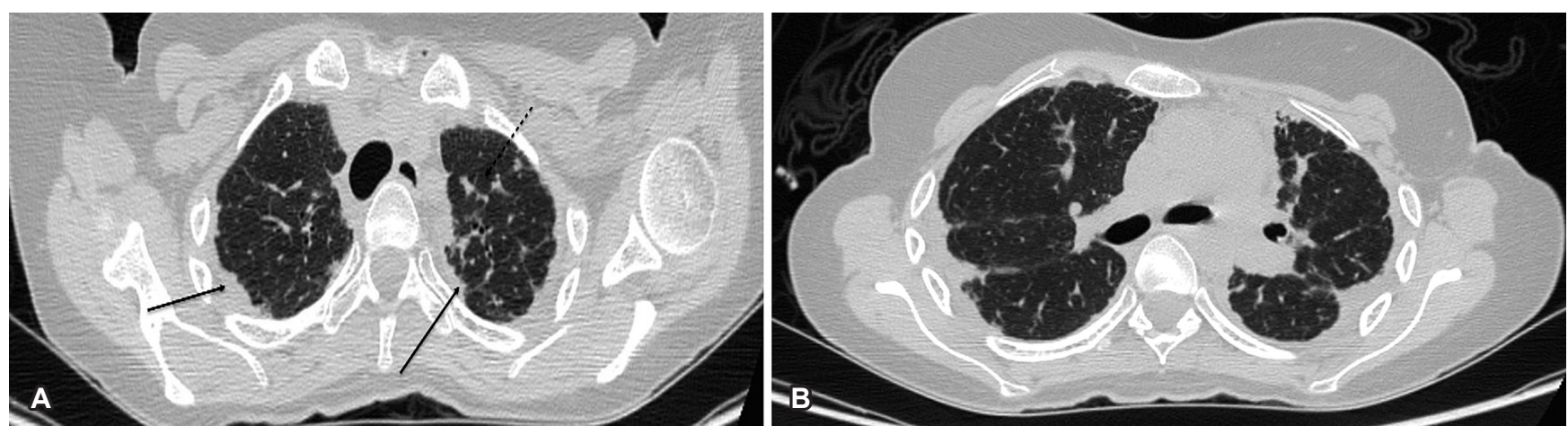

Figura 2. A. Tomografía de tórax de alta resolución, corte axial. Engrosamiento pleural (flechas) con engrosamiento de septos interlobulillares de predominio en los lóbulos superiores (flecha discontinua). B. Tomografía de tórax de alta resolución, corte axial. Engrosamiento pleural con consolidaciones subpleurales (flechas) de predominio en lóbulos superiores y distorsión de la arquitectura por cambios fibróticos (flecha discontinua). 
presencia de algunos granulomas pobremente conformados, en los cuales se identificaron algunos cristales de colesterol. Hubo engrosamiento de septos, que mostraron marcada congestión e infiltrado inflamatorio linfocitario con focos de antracosis. Algunos bronquios presentaron daño en su pared con fibrosis focal (Figura 3). La coloración tricrómica evidenció la fibrosis pleural y subpleural con extensión a septos (Figura 4). La coloración de Ziehl-Neelsen fue negativa para bacilos ácido alcohol resistentes (BAAR), y la plata metenamina negativa para hongos (Figura 5).

La paciente se valoró en junta médica multidisciplinaria (cirugía de tórax, radiología, neumología y patología), considerando cuadro clínico de fibroelastosis pleuroparenquimatosa en fase fibrótica, y requiriendo trasplante bipulmonar. Luego del procedimiento, la paciente presentó pobre reexpansión pulmonar, con de-
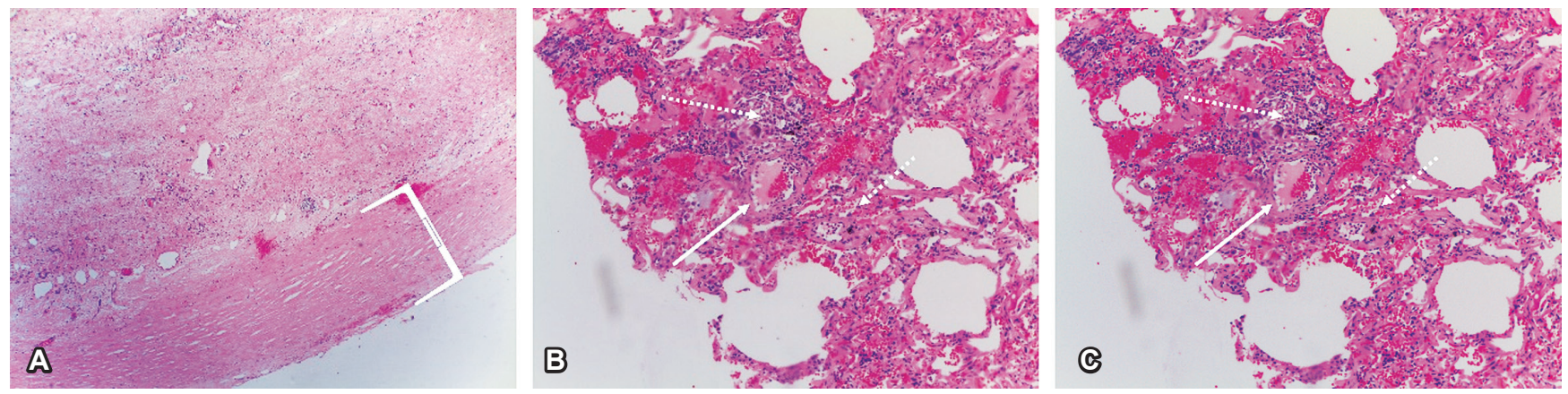

Figura 3. A. Técnica de coloración: hematoxilina eosina. Aumento 40x. Marcado engrosamiento pleural (corchete) con infiltrado inflamatorio linfocitario y fibrosis, el cual se extiende al parénquima cicatricial. B. Técnica de coloración: hematoxilina eosina. Aumento 200x. Infiltrado inflamatorio linfocitario y fibrosis (flechas discontinuas), el cual se extiende al parénquima cicatricial con presencia de algunos granulomas pobremente conformados, en los cuales se identifican algunos cristales de colesterol. Hay engrosamiento de septos, que muestran marcada congestión e infiltrado inflamatorio linfocitario con focos de antracosis (flechas discontinuas). Algunos bronquiales presentan daño en su pared con fibrosis focal. Luces alveolares con líquido de edema y macrófagos en su interior (flecha continua). C. Técnica de coloración: hematoxilina eosina. Aumento 400x. Granuloma pobremente conformado (recuadro), en el cual se identifican algunos cristales de colesterol. Existe engrosamiento de septos, que muestran marcada congestión e infiltrado inflamatorio linfocitario con focos de antracosis (flecha).

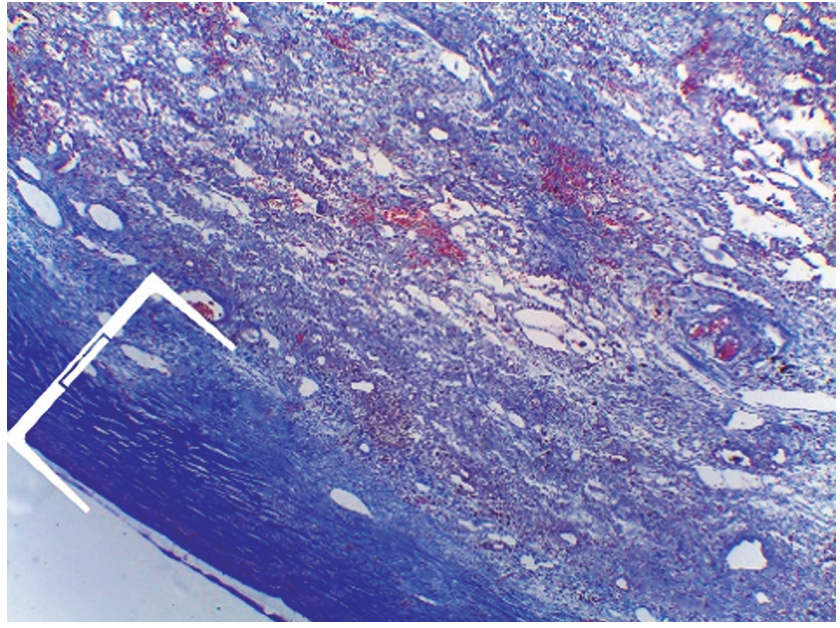

Figura 4. Técnica de coloración: tinción tricrómica (para observar colágeno y fibrosis). Aumento de 40x. Marcado engrosamiento por fibrosis pleural y subpleural (corchete), el cual se extiende a septos del parénquima cicatricial.

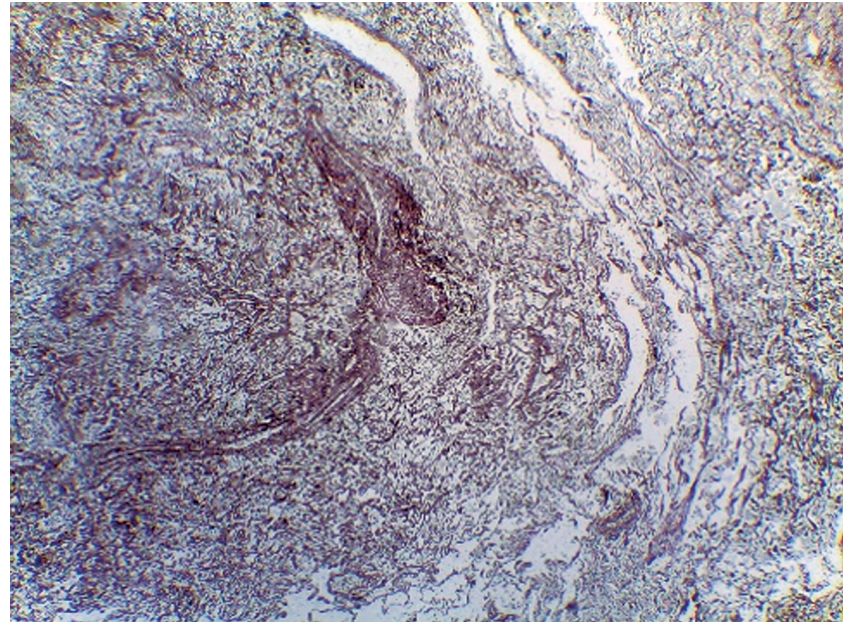

Figura 5. Técnica de coloración: plata metenamina negativa para hongos. Aumento 40x. 
saturación, somnolencia e hipercapnia; después indicó falla ventilatoria y falleció.

\section{Discusión}

La fibroelastosis pleuroparenquimatosa es una entidad rara que consiste en una forma de fibrosis que involucra la pleura y parénquima pulmonar subpleural, predominantemente de los lóbulos superiores. La progresión de la enfermedad ocurre en $60 \%$ de los pacientes, con muerte en $40 \%$ de los casos (6). Sus mecanismos patológicos y etiológicos son aún poco conocidos. La mayoría de las veces suele ser idiopática, aunque se cree que algunos casos ocurren en un contexto de enfermedad pulmonar familiar o genética, neumonitis por hipersensibilidad y otros casos han reportados asociación con trasplante de médula ósea, rechazo en pacientes con trasplante de pulmón, enfermedades vasculares del colágeno, enfermedades autoinmunes (artritis reumatoide, colitis ulcerativa y espondilitis anquilosante), quimioterapia, tabaquismo e incluso relacionada con infecciones respiratorias recurrentes, como Aspergillus, debido al proceso inflamatorio crónico asociado $(1,3,5,7,8)$.

La edad promedio de presentación es 53 años, con dos picos de aparición: uno a la tercera década y otro en la sexta década de la vida. En 45\% de los casos afecta al género masculino; sin embargo, en el pico de los pacientes más jóvenes hay cierta predominancia por el género femenino (5).

Existe variabilidad en la presentación clínica entre los pacientes reportados con fibroelastosis pleuroparenquimatosa idiopática: disnea, tos crónica, pérdida de peso, dolor torácico y neumotórax espontáneo $(1,5)$.

En cuanto al diagnóstico por imágenes, en la radiografía de tórax se evidencia cambios iniciales, como engrosamiento irregular de las porciones apicales de los pulmones; opacidades hiliares se pueden presentar con la progresión de la enfermedad. Con el tiempo, las opacidades reticulares y nodulares pueden aparecer en los segmentos superiores (5). En las etapas iniciales, la TACAR muestra opacidades reticulares y nódulos subpleurales en las regiones apicales, con mínimos cambios en las porciones medias e inferiores de los pulmones. Aun- que el compromiso es predominantemente subpleural, también puede encontrarse peribronquial; sin embargo, con la progresión de la enfermedad se presenta engrosamiento pleuroparenquimatoso asociado con fibrosis subyacente, elevación hiliar e importante pérdida de volumen, y distorsión de la arquitectura del parénquima pulmonar $(5,9)$. En estados avanzados se presentan bronquiectasias por tracción con múltiples bulas y quistes en los segmentos superiores $(1,5,7)$. Adicionalmente, los patrones de enfermedades pulmonares intersticiales pueden observarse en las otras partes del pulmón, como hallazgos de neumonía intersticial usual, neumonía intersticial no específica, entre otros (5).

Se debe enfatizar que la fibroelastosis pleuroparenquimatosa es una entidad que requiere un enfoque multidisciplinario que incluya al médico clínico, radiólogo y patólogo $(1,5)$. El diagnóstico definitivo se basa en las características histopatológicas de las zonas de fibrosis de la pleura visceral, fibrosis intraalveolar subpleural con una transición abrupta con el parénquima pulmonar normal, infiltrados linfoplasmocíticos escasos y focos fibroblásticos, deposición de fibras elásticas densas (elastosis septal alveolar) en la lesión pulmonar fibrosa subpleural y paredes alveolares, confirmando el diagnóstico $(5,10)$.

En la autopsia de la paciente, el examen macroscópico reveló consolidación pulmonar y fibrosis, que aparecieron más al extremo en los lóbulos pulmonares superiores en una distribución subpleural. Sin embargo, la microscopía reveló elastosis parenquimatosa presente en cada lóbulo.

En términos de diagnóstico diferencial por imagen se incluyen enfermedades intersticiales que se acompañen de fibrosis, como la neumonía intersticial usual, fibrosis pulmonar idiopática, neumonía intersticial no específica, asbestosis, enfermedad del tejido conectivo (especialmente espondilitis anquilosante), sarcoidosis fibrosante, enfermedad pulmonar inducida por medicamentos o radiación, y neumonitis por hipersensibilidad crónica (5). Sin embargo, el predominio apical subpleural y el engrosamiento pleural asociado generalmente son muy útiles para distinguir la fibroelastosis pleuroparenquimatosa idiopática de otras entidades, aunque los hallazgos por imagen de algunos casos de 
neumonitis por hipersensibilidad crónica pueden ser idénticos (6).

No hay un tratamiento específico para esta enfermedad, aproximaciones empíricas varían desde esteroides, inmunosupresores, $\mathrm{N}$-acetilcisteína, hasta el trasplante de pulmón con resultados y pronóstico variables $(1,2,7)$.

\section{Conclusión}

La fibroelastosis pleuroparenquimatosa es una entidad poco común y subdiagnosticada, con incidencia y prevalencia aún inciertas. Se relaciona con enfermedades autoinmunes, infecciones, medicamentos, trasplante de médula ósea o asociada con otros tipos de neumonías intersticiales, por lo que este diagnóstico debe considerarse en pacientes con fibrosis de distribución apical. Las opciones de tratamiento son limitadas y su pronóstico es variable; de aquí la importancia de diagnosticar esta entidad idealmente en un grupo interdisciplinario que incluya neumólogo, radiólogo y patólogo.

\section{Referencias}

1. Redondo MT, Melo N, Mota PC, Jesus JM, Moura CS, Guimaraes $\mathrm{S}$, et al. Idiopathic pleuroparenchymal fibroelastosis: a rare but increasingly recognized entity. Rev Port Pneumol. 2015;21(1):41-4. DOI: 10.1016/j.rppnen.2014.04.00.

2. Thangakunam B, Isaac BT, Christopher DJ, Burad D. Idiopathic pleuroparenchymal fibroelastosis - A rare idiopathic interstitial pneumonia. Respiratory medicine case reports. Respir Med Case Rep. 2015;17:8-11. DOI: 10.1016/j. rmcr.2015.11.004.
3. Rosenbaum JN, Butt YM, Johnson KA, Meyer K, Batra K, Kanne JP, et al. Pleuroparenchymal fibroelastosis: a pattern of chronic lung injury. Hum Pathol. 2015;46(1):137-46. DOI: 10.1016/j.humpath.2014.10.007.

4. Frankel SK, Cool CD, Lynch DA, Brown KK. Idiopathic pleuroparenchymal fibroelastosis: description of a novel clinicopathologic entity. Chest. 2004;126(6):2007-13. DOI: 10.1378/chest.126.6.2007.

5. Cheng SK, Chuah KL. Pleuroparenchymal fibroelastosis of the lung: a review. Arch Pathol Lab Med. 2016;140(8):84953. DOI: 10.5858/arpa.2015-0166-RS.

6. Sverzellati N, Lynch DA, Hansell DM, Johkoh T, King TE Jr, Travis WD. American Thoracic Society-European Respiratory Society classification of the idiopathic interstitial pneumonias: advances in knowledge since 2002. Radiographics. 2015;35(7):1849-71. DOI: 0.1148/rg.2015140334.

7. Hurtado EJ, González ML, Soto MM, Rueda FJ, Nadal FJ, Cantero AR. Idiopathic pleuroparenchymal fibroelastosis, a new idiopathic interstitial pneumonia: a case report. Chron Respir Dis. 2016;13(3):312-6. DOI: 10.1177/1479972316628529.

8. Yoshida Y, Nagata N, Tsuruta N, Kitasato Y, Wakamatsu K, Yoshimi M, et al. Heterogeneous clinical features in patients with pulmonary fibrosis showing histology of pleuroparenchymal fibroelastosis. Respir Investig. 2016;54(3):162-9. DOI: 10.1016/j.resinv.2015.11.002.

9. Kushima H, Hidaka K, Ishii H, Nakao A, On R, Kinoshita Y, et al. Two cases of pleuroparenchymal fibroelastosis diagnosed with transbronchial lung biopsy. Respir Med Case Rep. 2016;19:71-3. DOI: 10.1016/j.rmcr.2016.07.008.

10. Esteves C, Costa FR, Redondo MT, Moura CS, Guimaraes $\mathrm{S}$, Morais A, et al. Pleuroparenchymal fibroelastosis: role of high-resolution computed tomography (HRCT) and CTguided transthoracic core lung biopsy. Insights Imaging. 2016;7(1):155-62. DOI: 10.1007/s13244-015-0448-3. 